\title{
A Satellite's Temperature Field Reconstruction Method Based on Structural Feature
}

\author{
Na Chen ${ }^{1, a}$, Weiqing $\mathrm{Li}^{2, \mathrm{~b}}$, Pengxiang $\mathrm{Yi}^{3, \mathrm{c}}$ and Xinlong $\mathrm{MaO}^{4}$ \\ ${ }^{1}$ School of Computer Science \& Engineering, Nanjing University of Science \& Technology, Nanjing, \\ China \\ achenna255@163.com, ㄴi_weiqing@139.com, yipengxiang@foxmail.com
}

\begin{abstract}
Keywords: satellite, temperature field reconstruction, heat balance equation, visualization Abstract. Temperature field reconstruction of satellite is important for measuring temperature and monitoring the satellite's components. The reconstruction method of temperature field and the visualization technology in the satellite thermal control test were introduced. By analyzing typical internal structure of satellite and studying some different heat conduction structure, heat balance equation was established to find the main factors influencing the measuring points' temperature. For each heat conduction structure, the temperature field equation was reconstructed. A method of the temperature field reconstruction based on the satellite's structure was proposed. With the examination of typical heat transfer structures, the method was verified. The temperature field reconstruction method was used in a three-dimensional temperature visualization system and it worked fine.
\end{abstract}

\section{Introduction}

Thermal vacuum test was executed to simulate space environment on the ground and observe the satellite's working state, in order to validate the ability of satellite assembly under the thermal vacuum environment and expose the defects of components and materials, as well as the process, to eliminate early failure. Thermal vacuum test needs to monitor the temperature of the satellite, so the temperature field reconstruction and visual display is of great significance for the test [1].

Utilizing the known limited temperature data, the temperature field reconstruction restoral and reconstruct the distribution of temperature by calculation [2]. Chang Danhua et al of Yanshan University reconstructed the flame 3D temperature field by using area array CCD and introduced the method of outer contour to reconstruct the flame 3D temperature field [3]. Zhou Xian et al of electrical and information college of Xihua University proposed a 3D temperature field reconstruction algorithm based on the neural network radial basis function (RBF) [4]. To sum up, common methods of the target temperature field reconstruction are temperature field reconstruction based on temperature images, temperature field reconstruction based on acoustic measurement data, temperature field reconstruction based on optical information, and so on [5].

The accuracy of the methods described above is affected by the quantity of measuring points, the more measuring points and data are, the more accurate the method is. In satellite systems, due to the limited number of temperature measuring points, and without considering the characteristics of different components of the satellite, the above methods aren't precise enough. Based on of the internal structure features of the satellite, the following method used temperature data of existing measuring points inside satellite, calculated the temperature where didn't have measuring points, and then reconstructed the temperature field inside the satellite.

\section{Establishing Thermal Equilibrium Equation}

The instrument inside satellite is not in direct sunlight, which is affected by temperature of heat source itself, but also is associated with other surrounding facilities. Inside the satellite cover, we can analyze the thermal equilibrium relationship among measuring point units on the surface of the instrument and establish the thermal equilibrium equation.

Factors of affecting the temperature of measuring point unit: 
1) The heating of measuring point unit by other components inside the satellite's cover $Q_{1}$.

$$
Q=\alpha_{i} \cdot H_{0} \cdot A_{i}
$$

Where, $\alpha_{i}$ is the absorption rate of measuring point unit, $H_{0}$ is a constant of radiant heat source, $A$ is the area of measuring point unit.

2) The heat exchange between measuring point unit and adjacent unit $Q_{2}$.

$$
Q_{2}=\sum \frac{2 A_{j} K_{i} K_{j}}{K_{j} i_{i}+K_{i} r_{j}}\left(T_{i}-T_{j}\right)
$$

3) The heat radiation of measuring point unit $Q_{3}$.

$$
Q_{3}=b_{i} \cdot T_{i}^{4}
$$

Where, $b_{i}=\varepsilon_{i} \cdot \sigma \cdot A_{i}, \sigma$ is the Stefan-Boltzmann constant, the number of which is $5.67 * 10^{-3}$ $\mathrm{W} /\left(\mathrm{m}^{2} * \mathrm{~K}^{4}\right), \varepsilon_{i}$ is the thermal emissivity of measuring point unit.

4) The internal energy change of measuring point unit.

$$
Q_{4}=G C_{i}\left(d T_{i} / d t\right)
$$

Where, $C_{i}$ is the specific heat of unit i, $G$ is the quality of unit i, and $G=M A d_{i}, M$ is the density of unit $i, d_{i}$ is the thickness of unit $i$.

It can be obtained from the principle of heat conservation:

$$
Q+Q_{2}=Q_{3}+Q_{4}
$$

Adopt the difference method to linearize the equilibrium equation and conduct two simplifications. We can obtain the equilibrium equation and adjust the equation:

$$
\begin{aligned}
& 4 b_{i} T_{i}^{3}(t) T_{i}(T+\Delta t)+\frac{(\boldsymbol{c})_{i}}{\Delta t} T_{i}(t+\Delta t)+\left(\sum_{j \neq i} k_{j i}\right)\left(T_{i}(t+\Delta t)-T_{j}(t+\Delta t)\right) \\
& =3 b_{i} T_{i}^{4}(t)+\frac{(\boldsymbol{c})_{i}}{\Delta t} T_{i}(t)+\alpha_{i} \cdot H_{0} \cdot A
\end{aligned}
$$

The number of $k_{j i}$ is the equation is $\frac{2 A_{j} K_{i} K_{j}}{K_{j \Delta i}+K_{i \Delta j}}$.

From the principle of heat transfer, we can conclude that in the equation (6), the radiation factor of measuring point temperature change is $4 b_{i} T_{i}^{3}(t) T_{i}(T+\Delta t)$ and $3 b_{i} T_{i}^{4}(t)$, the internal energy change of measuring point is, the heat conductivity of measuring point unit temperature change is $\left(\sum_{j \neq i} k_{j i}\right)\left(T_{i}(t+\Delta t)-T_{j}(t+\Delta t)\right)$. The heating by the environment inside the satellite cover of measuring point unit temperature change is $\alpha_{i} \cdot H_{0} \cdot A_{i}$.

Here uses the method of the least square to solve to execute fitting calculation of coefficient in the equilibrium equation. The least square method searches the best matching function by minimizing the 
sum of error squares. The unknown coefficient can be calculated simply by the least square method, making the minimum sum of error squares between actual data and calculated data.

Transform the equilibrium equation in order to calculate:

$$
T_{i}(t+\Delta t)=\frac{3 A T_{i}^{4}(t)+A_{2} T_{i}(t)+A_{4}+\sum_{j \neq i} A_{3} T_{j}(t+\Delta t)}{4 A_{i} T_{i}^{3}(t)+A_{2}+n \cdot A_{3}}
$$

Where $\left(A_{1}, A_{2}, A_{3}, A_{4}\right)$ are corresponding to (radiation, internal energy change, heat conductivity, environmental thermal heat), $n$ is the amount of measuring point adjacent the measuring point.

As can be seen from the simplified equation factors influencing the temperature of a point $\operatorname{are}\left(A_{1}, A_{2}, A_{3}, A_{4}\right)$. Next analyze the typical structure type of the satellite inner components to find a method of calculating the temperature, and reconstruct the temperature field inside the satellite cover, and then make it visualized. Use the method of the least square to fit and calculate the value of $\left(A_{1}, A_{2}, A_{3}, A_{4}\right)$ in the equilibrium equation of every measuring point.

Make the square of the difference between actual value $T_{i}(t+\Delta t)^{\prime}$ and calculated value minimum as the best optimal judgment of the least square method.

$$
\varphi=\sum\left(T_{i}(t+\Delta t)^{\prime}-T_{i}(t+\Delta t)\right)^{2}
$$

Let $\varphi$ be the minimum, use Matlab to calculate the value of $\left(A_{1}, A_{2}, A_{3}, A_{4}\right)$.

$T_{j}(t+\Delta t)$ Calculated with the temperature value of the measuring point adjacent the measuring point. $T_{i}(t)$ Calculated with the current temperature value of the measuring point. $T_{i}(t+\Delta t)$ Calculated with the temperature value of the current measuring point in the next moment.

\section{The Classification of Satellite's Heat Transfer Structure}

When the satellite is operating in outer space, as in the microgravity and vacuum state, thermal convection disappears, so the main methods of heat transfer in space are conduction and thermal radiation.

Conduction. In this kind of structure conduction is the main heat transfer method. Heat is transferred rapidly between the contact sources[6]. The typical structure is heat pipe, which is a device depending on evaporation, condensation and circulation to transfer heat. The heat transfer performance is far better than the metal rod of the same size[7].

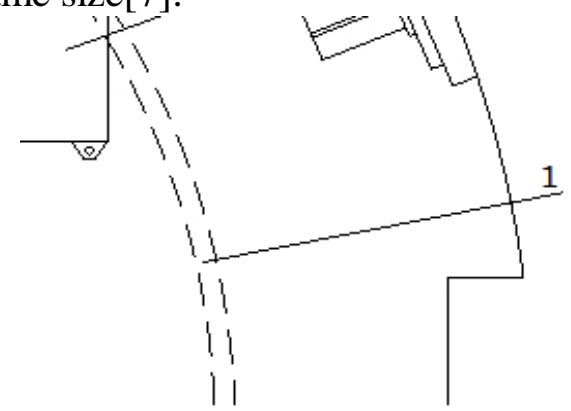

Fig.1 Schematic of Heat Pipe

As shown in Fig.1, the heat pipe is distributed on the back or sandwich of the instrument panel, bonded with the instrument panel, so that the heat can be transmitted from the instrument panel to the heat pipe, in order to the regulate the temperature to ensure the safety of the instrument.

Thermal Radiation. In this kind of structure thermal radiation is the main heat transfer method, absorbing or emitting heat by thermal radiation. 
The typical structure is satellite skin. Skin is commonly used to deal with heat that is emitting the heat through the larger coefficient of outer surface and avoiding absorbing the heat through the very low coefficient of inner surface. Meanwhile, in order to avoid the heat into the satellite interior by conduction, we also use a kind of insulation material with small thermal conductivity to separate from the internal structure of the skin. Therefore, thermal radiation is the main method of heat transfer in skin [8].

\section{Temperature Field Reconstruction of Typical Structure}

Racemic Component and Antenna Cylinder Structure. In Fig.2, measuring points 16 18 lie on the outer mask of the antenna cylinder structure. From the position we know measuring point 17 is between 16 and 18. Suppose that the temperature value of measuring point 17 is unknown, compare the calculated temperature value with the actual temperature and then verify the correctness.

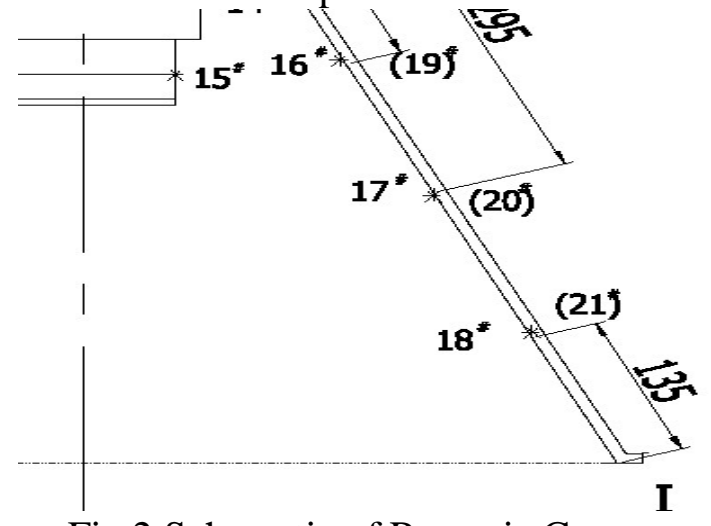

Fig.2 Schematic of Racemic Component and Antenna Cylinder Structure

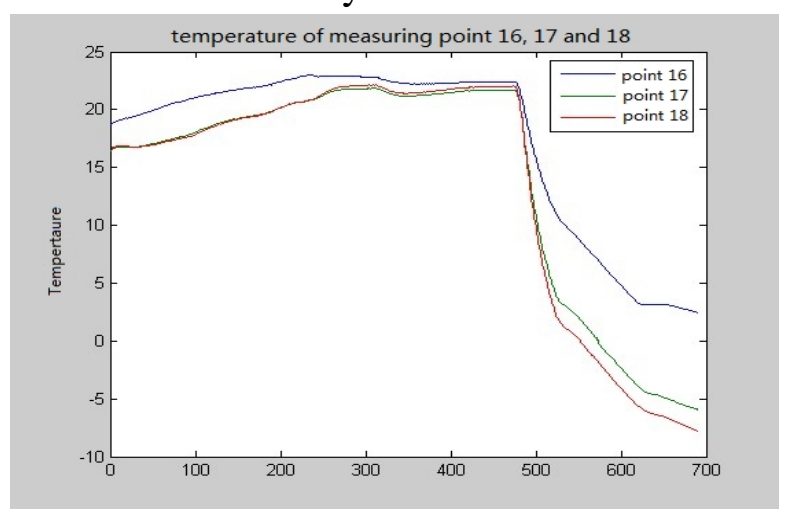

Fig.3 Temperature Value Curve 16 18 of Measuring Point

Firstly, observe the temperature value of measuring 16 and 18 from Fig.3. Secondly, figure out the coefficient of the thermal equilibrium equation according to the formula (6) and bring the temperature of adjacent point into calculation mutually. For example, regard the temperature of measuring point 18 as the temperature of adjacent point $T_{j}(t+\Delta t)$ of measuring point 16 and take it into calculation. So we can get the coefficient of the thermal equilibrium equation respectively: $\left(A, A_{2}, A_{3}, A_{4}\right)_{16}=(0.0001,-12.2765,1.0001,2.8947)$

$$
\left(A, A_{2}, A_{3}, A_{4}\right)_{18}=(0.0001,-13.8585,3.1856,26.0964) \text {. }
$$

By comparison we found the value of $\mathrm{A} 1$ and $\mathrm{A} 4$ in the equilibrium equation is differently, indicating that the factors of affecting the temperature are conductivity and environmental heat.

Because of measuring point 16 and 18 around 17, bring the temperature value of measuring point 16 and 18 as the temperature of adjacent point $T_{i}(t+\Delta t)$ into calculation to establish the thermal equilibrium equation.

$$
\begin{aligned}
& 4 A T_{17}^{3}(t) \cdot T_{17}(t+\Delta t)+A_{2} \cdot T_{17}(t+\Delta t)+A_{3} \cdot\left(T_{17}(t+\Delta t)-T_{16}(t+\Delta t)\right) \\
& +A_{3} \cdot\left(T_{17}(t+\Delta t)-T_{18}(t+\Delta t)\right)=3 A \cdot T_{17}^{4}(t)+A_{2} \cdot T_{17}(t)+A_{4}
\end{aligned}
$$

Due to measuring point 17 is between 16 and 18, when calculating we regard the initial average temperature of 16 and 18 as the initial temperature of 17 . The coefficient of measuring point 17 in the equilibrium equation is determined by the position of measuring point 16 and 18 . Measuring point 17 is between 16 and 18 , so the coefficient of measuring point 17 is:

$\left(\mathrm{A}_{1}\right)_{17}=\left(\mathrm{A}_{1}\right)_{16},\left(\mathrm{~A}_{2}\right)_{17}=\left(\mathrm{A}_{2}\right)_{16},\left(\mathrm{~A}_{3}\right)_{17}=\left(\left(\mathrm{A}_{3}\right)_{16}+\left(\mathrm{A}_{3}\right)_{18}\right) / 2,\left(\mathrm{~A}_{4}\right)_{17}=\left(\left(\mathrm{A}_{4}\right)_{16}+\left(\mathrm{A}_{4}\right)_{18}\right) / 2$.

Take it into equilibrium equation and calculate the temperature of measuring point. The value is shown in Fig.4: 


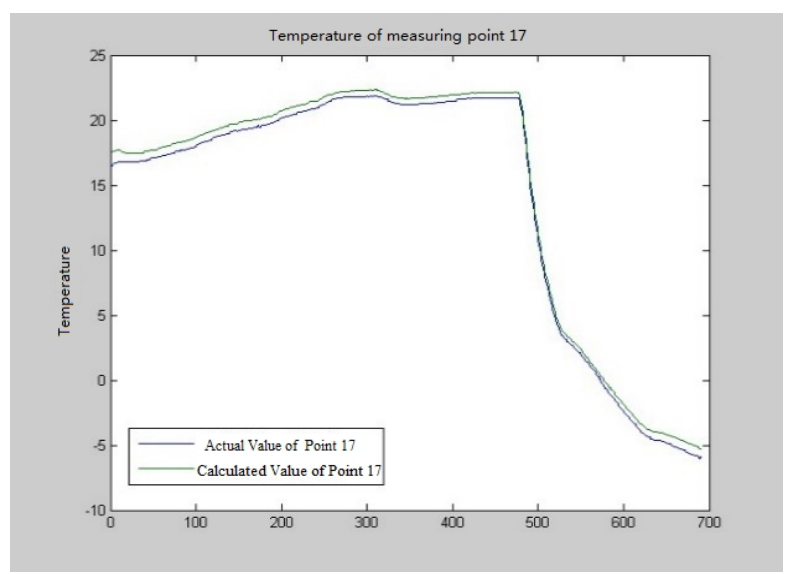

Fig.4 Comparison of Actual Value and Calculated Value of Measuring Point 17

We described the reasonableness of the temperature calculation method by calculating the average temperature error and the maximum temperature error:

$$
\mathrm{K}=\frac{\sum_{i=1}^{n}\left(T S_{i}-T C_{i}\right)}{n}
$$

Where, $\mathrm{K}$ is the average temperature error, TS is the calculated temperature value of the measuring point, TC is the actual temperature value of the measuring point. The temperature difference of satellite in space can reach 200-300 degrees [9]. By calculation the average temperature error between the actual value and calculated value of measuring point is $\mathrm{K}=0.799$ Degree, the maximum temperature difference is 1.001 Degree. Compare the average error with the temperature difference in space and expect the result to be more accurate.

The Instruments on the Instrument Board.

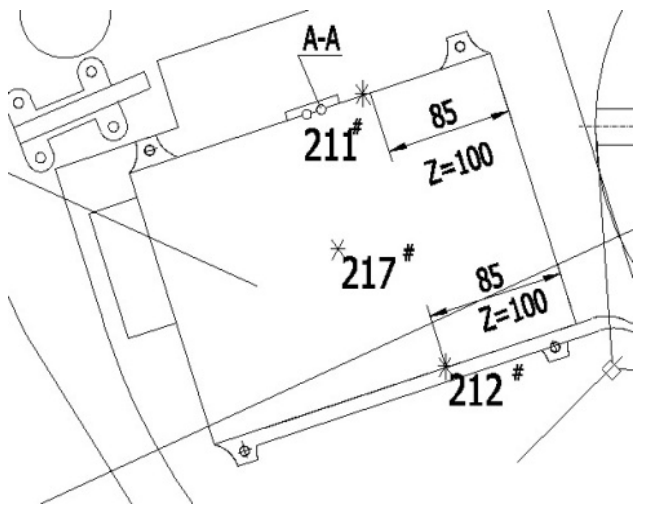

Fig.5 Secondary Power Source on the Instrument Board

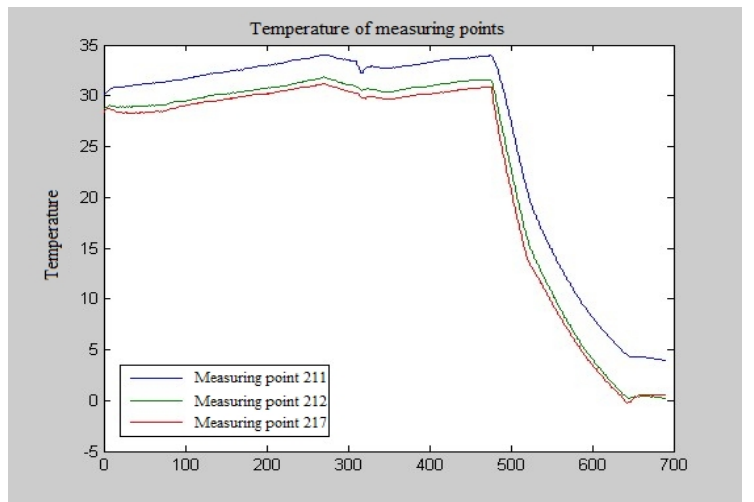

Fig.6 Temperature Value Curve of Measuring Point 211, 212 and 217

Firstly, we get the temperature of measuring points 211, 212 and 217 from Fig.5.We can get the temperature of measuring point 212 by that of measuring point 211 and 217 in Fig.6. By establishing thermal equilibrium equation of measuring points 211 and 217 , getting the factors that affect the temperature of measuring points.

The thermal equilibrium equation of measuring point 211:

$$
\begin{aligned}
& 4 \cdot A T_{211}^{3}(\mathrm{t}) \cdot T_{211}(t+\Delta t)+A_{2} \cdot T_{211}(t+\Delta t)+A_{3} \cdot\left[T_{211}(t+\Delta t)-T_{217}(t+\Delta t)\right] \\
& =3 A \cdot T_{211}^{4}(\mathrm{t})+A_{2} \cdot T_{211}(t)+A_{4}
\end{aligned}
$$

We can calculate the value: $\left(A, A_{2}, A_{3}, A_{4}\right)_{211}=(0.0000,-9.1899,1.3071,-3.8812)$.

The thermal equilibrium equation of measuring point 217 : 


$$
\begin{aligned}
& 4 \cdot A T_{217}^{3}(\mathrm{t}) \cdot T_{217}(t+\Delta t)+A_{2} \cdot T_{217}(t+\Delta t)+A_{3} \cdot\left[T_{217}(t+\Delta t)-T_{211}(t+\Delta t)\right] \\
& =3 \cdot A T_{217}^{4}(\mathrm{t})+A_{2} \cdot T_{217}(t)+A_{4}
\end{aligned}
$$

We can get calculate the value $\left(A, A_{2}, A_{3}, A_{4}\right)_{217}=(0.0001,-46.1614,2.7489,-16.8935)$.

From the two groups of value $\left(A_{1}, A_{2}, A_{3}, A_{4}\right)$ we have calculated, we can find that four values of $\mathrm{A}$ are different. Measuring point 211, 212 and 217 are the measuring points on the secondary power sources. Secondary power sources are on the instrument board, on which there has a lot of satellite-borne instruments. Each kind of satellite-borne instruments have their own thermal environment, so we have to analyze the factors that influence the temperature of measuring points depending on the concrete conditions.

Measuring point 211 locates on the heat pipe of the secondary power source, and is for heat dissipation. Measuring point 212 and 217 are parts of the main body of secondary power source's shell, so their factors that influence their temperature are similar. Therefore, we can calculate $\left(A_{1}, A_{2}, A_{3}, A_{4}\right)_{212}$ by this way:

$$
\left(A, A_{2}, A_{3}, A_{4}\right)_{212}=\frac{a\left(A, A_{2}, A_{3}, A_{4}\right)_{211}+b\left(A, A_{2}, A_{3}, A_{4}\right)_{217}}{a+b}
$$

Here, $a$ and $b$ are the weight of how much measuring point 211 and 217 influence the temperature of measuring point 212. Depending on the distance of the measuring points, we can use 1 as value of a and 2 as value of $b$ to calculate the value of $\left(A, A_{2}, A_{3}, A_{4}\right)_{212}$.

The thermal equilibrium equation of measuring point 212 :

$$
\begin{aligned}
& \text { 4. } A T_{211}^{3}(\mathrm{t}) \cdot T_{211}(t+\Delta t)+A_{2} \cdot T_{211}(t+\Delta t)+A_{3} \cdot\left[T_{211}(t+\Delta t)-T_{217}(t+\Delta t)\right] \\
& =3 \cdot A T_{211}^{4}(\mathrm{t})+A_{2} \cdot T_{211}(t)+A_{4}
\end{aligned}
$$

Introducing the value of $\left(A, A_{2}, A_{3}, A_{4}\right)_{212}$ to equation (14) and setting the initial temperature of measuring point 212 as the initial value, we can finally get the figure of calculated value and actual value of measuring point 212 in Fig. 7.

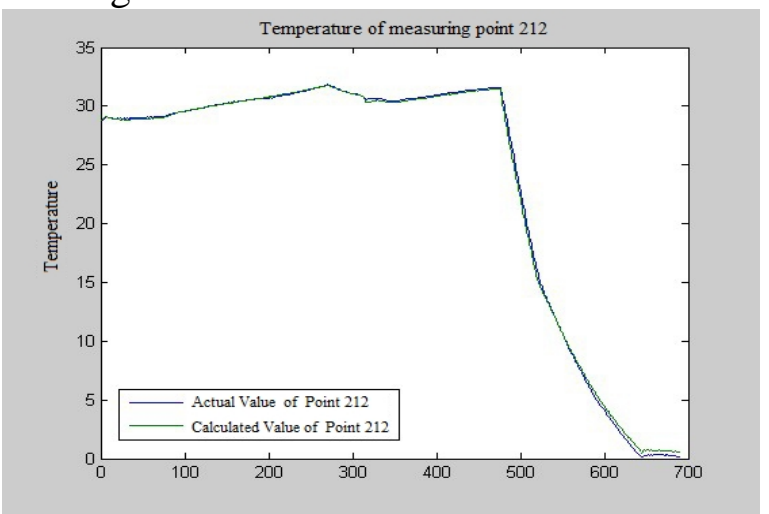

Fig.7 Comparison of Actual Value and Calculated Value of Measuring Point 212

The average temperature error between the actual value and calculated value of measuring point 212 is 0.225 Degree, and the maximum temperature difference is 0.496 Degree. So the result is reasonable.

\section{Visualization of Satellite Temperature Field}

The method is applied to 3D satellite temperature display system. The system uses OpenSceneGraph as the $3 \mathrm{D}$ engine and reconstructs the satellite temperature field with the help of the method mentioned in 
this paper. We can utilize the program to modify the color on the model surface to represent different temperature, which can achieve the purpose of monitoring the satellite temperature.

\section{Conclusion}

This paper presents a method of the satellite temperature field reconstruction based on structure feature. This method takes into account the structure feature of different components of the satellite and reconstructs temperature field according to the temperature of limited measuring points.

According to the experimental results, it has certain rationality. If every point in the 3D model is calculated with the reconstruction method mentioned in the paper, the computation will be very large. Therefore in practical application the temperature field reconstruction method is only applied to the key components and measuring point.It's required to establish the thermal equilibrium equation for different components and figure out the temperature value of measuring point whose computation may be large. There's certainly space for promotion in the arithmetic performance.

Taking into account the temperature feature of different satellite components, we should make a concrete analysis of each specific question. The algorithm described in this paper mainly aims at the satellite with certain limitations. The method described in this paper has significance to promote for the reconstruction of temperature field.

\section{References}

[1] M. Azzouzi, and F. Neri, An Introduction to the Special Issue on Advanced Control of Energy Systems. WSEAS Transactions on Power Systems, Vol. 8, No. 3, 2013, p. 103.

[2] C. Danhua, Y. Boran, and X. Chuangjie, An Experimental Study on Visualization of 3d Temperature Fields in Flames. Electronic Technology. Vol. 45, No. 3, 2008, pp. 66- 68.

[3] I. Pollini, Satellite Structure in the Photoelectron Spectra of Transition-metal Compounds. Philosophical Magazine B, Vol. 81, No. 12, 2001, pp. 2055-2070.

[4] S. R. Kumar, S. G. Barve, and K. B. Narayana Thermal Design and In-orbit Performance of ANUSAT. Journal of Spacecraft Technology, Vol. 20, No. 2, 2010, pp. 37-46.

[5] S. Feng, Z. Huaichun, H. Shudong, L. Jun, Z. Chuguang, Reconstruction of Temperature Profiles in Two-dimensional Furnaces from Radiation Image by Solving Inverse Radiative Heat Transfer Problem. Proceedings of the CSEE. Vol. 19, No. 10, 1999, pp. 1-5.

[6] Z. Zhanrong, Z. Youzhang, Z. Qinghua, Z. Yunfang, A Method of Reconstructing Temperature Field Based on Abel Transform. Laser Journal, Vol. 30, No. 3, 2009, pp. 18-19.

[7] W. Jiajun, Design and Analysis of Satellite Structure. Beijing: China Astronautic Publishing House. 2004.

[8] W. Chen, Experimental Investigations of Heat Transfer Characteristics of Flat Plate Heat Pipe for Electronic Cooling. PhD Thesis. Beijing University of Technology, 2013.

[9] Dombrovsky L A, Baillis D, Randrianalisoa J H.Some physical models used to identify and analyze infrared radiative properties of semi-transparent dispersed materials. Journal of Spectroscopy Dynamics, 2011, 1(1): 1-20. 